\title{
Artificial Intelligence as a Public Service: Learning from Amsterdam and Helsinki
}

\author{
Luciano Floridi $^{1,2}$
}

Received: 18 October 2020 / Accepted: 18 October 2020 /Published online: 21 October 2020

(C) Springer Nature B.V. 2020

\section{Introduction}

On September 28, 2020, at the Next Generation Internet Summit, Helsinki and Amsterdam announced the launch of their open AI registers. They are the first cities to offer such a service in the world (City of Helsinki 2020). The AI registers describe what, where, and how AI applications are being used in the two municipalities; which datasets were used for training purposes; how algorithms were assessed for potential bias or risks; and how humans use the AI services. The registers also offer a feedback channel, which is meant to enable more participation, with information about the city department and the person responsible for the AI service. The goal is to make the use of urban AI solutions as responsible, transparent, and secure as other local government activities, to improve services and citizens' experiences.

The AI registers are currently being populated. Anyone can check them. At the time of writing, there are 5 AI services available in the Helsinki AI Register ${ }^{1}$ and 3 in the Amsterdam AI Register. ${ }^{2}$ The plan is eventually to have all the cities' AI services listed in the registers. At the moment, eight services are not many, but, despite their still limited number, the overall project is extremely interesting for several reasons, and one can learn a few lessons from it. Let us see them.

\section{Why the Project Is Interesting}

\subsection{Normal Al}

Despite all the pointless but very distracting speculations about nasty robots, singularity, superintelligence, and other sci-fi dystopian stories, the sort of AI appearing in the

\footnotetext{
${ }^{1}$ https://ai.hel.fi/en/ai-register/

${ }^{2}$ https://algoritmeregister.amsterdam.nl/en/ai-register/

Luciano Floridi

luciano.floridi@oii.ox.ac.uk

1 Oxford Internet Institute, University of Oxford, 1 St Giles, Oxford OX1 3JS, UK

2 The Alan Turing Institute, 96 Euston Road, London NW1 2DB, UK
} 
registers is, as expected, just ordinary digital technology, that deals with issues such as parking or reporting maintenance problems. This is how the project describes AI:

Artificial intelligence refers to systems that observe the environment and process information in order to achieve, without guidance, the objectives assigned to them. In the case of city services, artificial intelligence may, for example, recommend books suitable for the library's customers or issue instructions for those consulting, for instance, childbirth \& maternity counselling. Artificial intelligence also seeks, for example, easy and rapid access to information, identification of objects or people, appropriate recommendations or more efficient traffic control. At the same time, it should be borne in mind that artificial intelligence does not function in a vacuum or on its own. A person ultimately responsible for the operation of artificial intelligence is always needed to teach and supervise the machine. (City of Helsinki 2020)

It is a satisfactory description that anyone can understand. Note how AI is understood as a means to an end, as it should, not a crazy robot with a mind of its own, and hence the reference to a human supervisory role and human responsibility, which is good common sense. The project shows that $\mathrm{AI}$ is not a marriage between engineered artefacts and some kind of human-like or even superhuman intelligence, but a divorce between the ability to solve a problem successfully in view of a goal and the need to be intelligent in doing so (Floridi 2019). With enough data, statistics, and computational power, one can do without intelligence. Indeed, it is becoming easier to understand intelligence as whatever enables an agent to perform successfully a task that otherwise would require immense quantity of data, sophisticated statistical tools, huge computational power, and much more energy. The sooner we realize this, the earlier we shall be able to develop many valuable ways of deploying AI systems without any fear that Terminator might be coming.

\subsection{The "Utility-Fication" of Al as a Public Service}

Following the "normalization" of AI, it is interesting to see how the project presents AI as just another utility. AI is increasingly offered as a service, or AIaaS (Newman 2020), especially in the case of machine learning and natural language processing capabilities. Yet AIasS is a bit of an understatement, to say the least, since the truth is that, contrary to gas or water, AI is a new form of mindless agency into which one can tap to deal with problems that otherwise would require human intelligence and perhaps a huge (sometimes unfeasible) amount of other resources, like time. At the same time, part of the value of the project seems to lie also in the recognition that AI as a "utility" is a great means to deal with increasingly complex, urban environments. As the population of the world moves to live more and more in megacities, the latter may not become "smart", but they can certainly be managed much more intelligently by using AI systems that provide more effective and efficient services, in ways that are open and transparent to public scrutiny and feedback.

\subsection{Al as Part of the (Urban) Environment}

In many cities, not just Helsinki and Amsterdam, people are already interacting daily through, or with, AI applications. AI solutions are indistinguishably mixed with 
analogue and other digital components. This infosphere (Floridi 2014a), populated by new forms of artificial agency and inhabited by citizens who live "onlife" (Floridi $2014 b$ ), is an everyday and growing reality. So, another valuable aspect of the project consists in raising awareness about the normal, common, utility-like use of AI systems in urban environments and in city automation, and showing how our world is increasingly dependent on digital solutions.

\subsection{Al Ethics}

There is even more to like in the ethical approach shared by the project. The aforementioned dependence comes with potential ethical challenges. So this is the official statement about the ethics of AI:

Human-centred AI is built on open and ethically sustainable utilisation of technology and AI. The objective of Helsinki and Amsterdam is that artificial intelligence in public services must operate on the same principles of responsibility, transparency and security as other activities of the city. (City of Helsinki 2020)

Admittedly, it is a pity that the project falls for the "human-centric" rhetoric. After four revolutions that have decentralized humanity from the universe (Copernicus), the animal kingdom (Darwin), the space of reason (Freud), and the infosphere (Turing), ${ }^{3}$ we should have learnt that trying to place humanity back at the centre of something is often a way of reintroducing some kind of chauvinism, and risking to treat the rest of the planet as something at the service of humanity, not to be respected and taken care of, but merely exploited. However, it seems clear that this is not what the project means. The invitation is rather that of putting digital technology, and AI in particular, at the service of humanity. The next step would be to see humanity as the caretaker of all realities and habitats (Floridi 2013). At the same time, after all the fuss about so many codes, declarations, manifestos, lists of principles, and frameworks (Floridi and Cowls 2019), framing AI within the already available good practices of urban policies is refreshing. Normal, utility-like, city-embedded AI public services are expected to fit within pre-existing frameworks that already provide well-tested and reliable guidelines for their governance. This also suggests that such framing may be reproducible wherever city governance is done properly, as I shall argue below. ${ }^{4}$

\subsection{Local and Bottom-Up Approach to Al as a Public Service}

The project shows daily realities and experiences in the life (or rather onlife) of ordinary citizens. This "AI on the ground" is where real and positive changes can happen more likely, in ways not dissimilar from what is happening to green policies that seek to contrast climate change or pollution. For example, in the EU, the AI4Cities project, ${ }^{5}$ funded by the EU's Horizon 2020 research and innovation programme, links 6

\footnotetext{
${ }^{3}$ For an interpretation, see Floridi (2014a).

${ }^{4}$ For a list of cities supporting digital rights, see "Cities for Digital Rights": https://citiesfordigitalrights.org/.

${ }^{5}$ https://ai4cities.eu/
} 
European cities and regions looking for AI solutions on mobility and energy to accelerate carbon neutrality. Interestingly, two of them are Amsterdam and Helsinki (the others are Copenhagen, Paris Region, Stavanger, and Tallinn). In the USA, we know that real changes in terms of environmental sustainability are fostered by cities and some States, not by the federal government. This local and bottom-up approach enables a higher degree of variety and flexibility of solutions. Admittedly, the approach may be risky, if it does not lead to cooperation, because there are systemic and global challenges as well as opportunities that require the right size of "agency" to be deal with, think for example of the current pandemic. However, the fact that the project involves two cities belonging to two different EU members states already shows that cooperation is possible and welcome. It should also be fostered to ensure that other cities will join soon. London, for example, is developing an Emerging Technologies Charter:

The Charter will consider areas such as ways of working, including engagement with the public and other relevant bodies when trialling new technology; the collection, use and sharing of data from emerging technologies; how inclusion and accessibility can be ensured; and privacy and security. (Wray 2020)

Other cities have compatible or complementary initiatives: from Boston's Smart Cities Playbook $^{6}$ to New York's creation of an Algorithms Management and Policy Officer ${ }^{7}$ to oversee algorithmic decision-making (following the creation of an automated decision systems task force in 2017 and a critical report), and from San Francisco's Emerging Technology Board ${ }^{8}$ to Barcelona's Ethical Digital Standards. ${ }^{9}$ In short, an international, city-based, and city-led movement in support of AI would be a welcome development. Even if at the moment cities are not yet fully ready for the disruptions that AI may bring, European cities seem to be well placed, according to the Global Cities AI Readiness Index. ${ }^{10}$ And more than 40 important cities are members of the Cities Coalition for Digital Rights, ${ }^{11}$ the joint initiative launched by Amsterdam, Barcelona, and New York City with the support of UN-Habitat, EUROCITIES, CGLU to support digital rights: They could easily expand their activities to promote AI-based public services that are both sustainable and socially preferable.

\subsection{Scalability and Portability}

The previous points highlight two other positive features of the project: its local scalability and its geographical portability. We saw that other services can easily be added, leading to incremental refinements, and I just argued that other cities could also join. The value of such scalability and portability is that much can be learnt and shared, in terms of best technical solutions, lessons learnt, best practices, avoidable opportunity costs or wasted resources, citizens' feedback, and so forth. In particular, if cities can

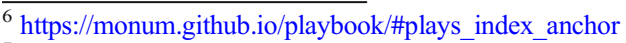

${ }^{7} \mathrm{https} / / / \mathrm{www} 1$. nyc.gov/site/ampo/index.page

${ }^{8}$ https://emergingtech.sfgov.org/

${ }^{9} \mathrm{https} / /$ www.barcelona.cat/digitalstandards/en/init/0.1/index.html

${ }^{10} \mathrm{https}$ //www.oliverwymanforum.com/city-readiness/global-cities-ai-readiness-index-2019.html

${ }^{11} \mathrm{https} / /$ citiesfordigitalrights.org/cities
} 
speak to each other about which kind of AI works well, when, for what, and for whom, many trials and errors could be avoided, and resources could be used more effectively and efficiently.

\subsection{Private and Public Partnership}

The project is implemented in partnership with Saidot, a Finnish company that specializes in digital technologies and services that support openness, transparency, and explicability of artificial intelligence and smart applications. This is a fruitful strategy, because city administrators can have detailed and high-quality knowledge about AI local services, their usability, value, and so forth, but often lack the technical expertise to translate this information into a public service.

\section{8 "Trust Through Transparency"}

Saidot advocates openness and transparency as ways of building trust among citizens about AI and other digital solutions. This is a sensible approach, but it is improvable and it may be insufficient. Trust also requires accountability and may also be reinforced by opportunities to participate in the early stages of the decision process that leads to the deployment of an AI public service, rather than just at the end, with feedback only about what is made available. So feedback should have some real teeth (accountability), and AI services may also need to be open in terms of co-design, both at the stage of planning and development and at the stage of deployment, once an AI public service is delivered. "Check and trust" should be complemented by a "co-design and co-own" the AI solutions adopted.

\subsection{Humans Watching Al}

There is something very positive about the openness and transparency promoted by the project. Although I just argued that such features may be insufficient to build trust, they have the side effect of creating a culture in which it is the human users who can watch and hence determine the behaviour of AI services. It does not matter whether anyone actually will check the registers. The fact that someone may be watching is sufficient to make a positive difference in terms of what should and should not be done to begin with, when it comes to using AI as a public service.

\section{Conclusion}

I hope that the experiment will be a success. But even if it does not, it still shows a very good way in which AI could be implemented as a public service, thus indicating a fruitful strategy that societies could and should pursue. AI is an extraordinary technology that can be of huge help in handling the increasing complexity of our world and the many challenges we are facing, including climate change, social justice, and public health. Harnessing it intelligently at the urban level is a smart move. It will remain a right move for the foreseeable future. 


\section{References}

City of Helsinki. (2020, 28 September). Helsinki and Amsterdam first cities in the world to launch open AI register. https:/news.cision.com/fi/city-of-helsinki/r/helsinki-and-amsterdam-first-cities-in-the-world-tolaunch-open-ai-register,c3204076.

Floridi, L. (2013). The ethics of information. Oxford: Oxford University Press.

Floridi, L. (2014a). The fourth revolution - how the Infosphere is reshaping human reality. Oxford: Oxford University Press.

Floridi, L. (Ed.). (2014b). The Onlife manifesto - being human in a Hyperconnected era. New York: Springer. Floridi, L. (2019). What the near future of artificial intelligence could be. Philosophy \& Technology, 32(1), 115. https://doi.org/10.1007/s13347-019-00345-y.

Floridi, L., \& Cowls, J. (2019). A unified framework of five principles for AI in society. Harvard Data Science Review, 1(1).

Newman, D. (2020, 7 January). Why AI as a service will take off in 2020. Forbes.

Wray, S. (2020, 30 July). London charter to set the ground rules for emerging technology. CitiesToday.

Publisher's Note Springer Nature remains neutral with regard to jurisdictional claims in published maps and institutional affiliations. 